\title{
FERMETURE D'USINE ET SANTÉ: ANALYSES DE SÉRIES CHRONOLOGIQUES CHEZ LES FEMMES DE 30 À 39 ANS DE LA RÉGION SEPT-ILES/PORT CARTIER
}

\author{
CAMIL BOUCHARD \\ Université du Québec à Montréal \\ ROBERT PERREAULT \\ Hôpital Général de Montréal
}

\section{RÉSUMÉ}

L'étude s'intéresse à l'impact de la fermeture de l'usine Rayonnier-Québec de Port-Cartier sur la fréquence des actes médicaux poses avant et aprés la fermeture sur une période cinq ans. La méthodologie employée permet de suggèrer l'existence d'un lien spécifique entre la fermeture de l'usine et une augmentation des examens complets chez le groupe de femmes de $30-39$ ans dans cette région.

La plupart des études rétrospectives concluent à une relation positive entre chômage et hospitalisations psychiatriques (Brenner, 1973), suicides et homicides (Vigherdous, 1977, 1978; Vigherdous et Fishman, 1978 in Adams, 1981), troubles physiologiques divers (Brenner, 1978), troubles cardiaques et morbiditế psychiatrique (Adams, 1981). Plus récemment les études prospectives de Dooley ct Catalano (1980) mettaient en évidence une felation entre la diminution de la force de travail et l'augmentation ultérieure de la fréquence des symptômes psychophysiologiques chez la population. Steinberg, Dooley et Catalano (1981) établissaient un lien spécifique entre l'augmentation du chômage et des matuvais traitements envers les enfants. De façon gènérale, malgré quelques résultats discordants et l'absence de modeles explicatifs clairs, la relation entre chômage et

L'équipe disposait d'un budget permettant the analyse partielle des séries chronologiques par traitements statistiques. Nous en sommes redevables au Fond Institutionnel de Recherche de l'Université de Montréal, (CAFIR). problèmes comportementaux et de santé semble bien établie,

La présente étude $s$ intéresse à l'impact présumé d'un changement brutal de l'économie d'une région sur l'évolution mensuelle de la fréquence des actes médicaux enregistrés. Plus spécifiquement, l'étude entend vérifier l'effet potentiel de la fermeture de I'usine Rayonnier-Québec de Port-Cartier en procédant à l'examen de la fréquence des actes médicaux posés avant et après la fermeture sur une période cinq ans. La fermeture de l'usine survint le 12 septembre 1979, après une grève de plus de trois mois de ses 1500 employés. A ce moment, PortCartier et la région (Clark City et Sept-lles) comptaient environ 40000 habitants.

\section{MÉTHODOLOGIE: CONSIDÉRATIONS PRÉLIMINAIRES}

Les ressources financières de notre équipe de recherche contraignaient au seul recours d'une méthodologie rétrospective permettant le traitement des données déjà disponibles. Les banques de données requises, pour fins d'analyses statistique de séries chronologi- 
ques, devaient être complètes, systématiques, étalées par intervalles mensuels sur une période relativement longue, et être disponibles au public. Seules les banques de la Régie de l'Assurance-Maladie du Québec (RAMQ) répondaient à ces critères. Aucune banque n'était disponible en ce qui a trait à un recensement des problématiques psychosociales, (problèmes de comportements individuels, violence familiale ou sociale, séparations, divorces, etc.). Une enquête auprès d'informateurs-clés permit, à cet égard, l'identification qualitative et nonsystématique de certains phénomènes; cette enquête a fait l'object d'une production vidéo* et certaines observations seront évoquées en discussion. L'essentiel du présent travail porte donc sur l'analyse des variations de fréquences d'actes médicaux posés dans ta région Sept-lles/Port-Cartier. Cette analyse comporte deux étapes: examen visuel de séries chronologiques partielles et, traitement statistique de séries chronologiques complètes par la technique de Box-Jenkins.

\section{EXAMEN VISUEL DES SÉRIES CHRONOLOGIQUES PARTIELLES}

Cette première étape ne pouvait viser à un traitement statistique des données; en effet, en mai 1980, les séries disponibles s'interrompaient à janvier 1980 , soit seulement trois mois après la fermeture. La technique BoxJenkins demande une série ultérieure à l'évenement beaucoup plus longue. La série de données comprenait donc, outre certaines catégories diagnostique spécifiques, les relevés mensuels du nombre d'actes médicaux posés pour les hommes et les femmes par strates d'âge durant les années 1977, 1978 et 1979 (Bouchard et Perreault. 1981).

L'examen visuel de ces données permet d'identifier une configuration redondante: augmentation du nombre d'actes en hiver,

* Ce vidéo s'intitule "Ad Portum Faliciter" et on peut en demander copie auprès des auteurs pour la somme de $\$ 25.00$. diminution en été. Une première observation s'impose alors que la fréquence maximale d'actes apparaît en novembre pour les annêes 1977 et 1978, e'est en octobre, soit 6 mois suj. vant la fermeture de l'usine, qu'elle prend place en 1979. Deuxième observation, cette bouffée prématurée est le fait de la popula. tion féminine. Enfin, troisième constatation, un examen détaillé des catégories de données (nature des actes médicaux posés et diagnostics) amène à constater que cette par. ticularité s'applique quasi uniquement aи groupe des 30-39 ans et seulement dans la catégorie des "examens physiques complets" (figure 1).

\section{TRAITEMENTS STATISTIQUES DES SÉRIES CHRONOLOGIQUES}

Théoriquement, la technique de BoxJenkins permet (1) d'identifier, le cas échéant, la présence d'une configuration redondante ("pattern") dans les données; (2) d'en dégager les paramètres; (3) d'en délester les donnees; (4) pour permettre l'analyse des scores résiduels et (5) de procéder, de la soric, à la reconnaissance de points qui se distingueraient du "built on," si l'on veut, des intercorrélations non significatives de la matrice des résiduels. En d'autres mots, ta technique permet de vérifier si la bouffée observée se différencie des fluctuations normalement présentes dans les données, et si elle coinncide avec l'ćvénement (fermeture) at l'étude. Cependant, dans l'affirmative, il faut encore écarter l'hypothese que cette bouffée exceptionnelle colncidant avec la fermeture puisse dépendre d'un autre événement intra-régionale ou supra-régional. Pour ce qui est de l'intra-régional, aucun événe ment majeur (grève, "lock-out," vaccina-

- Cette catégorie est la plus volumineuse purmi les actes posés. Il faut également notef qu'un examen physique complet est souvent pratiqué (1) chez un nouveau patient et (2) dans le cas de plaintes diffuses, ce qui fait di sens dans le contexte d'un changement bruts de l'économie. 
Figure 1

30-39 ANS

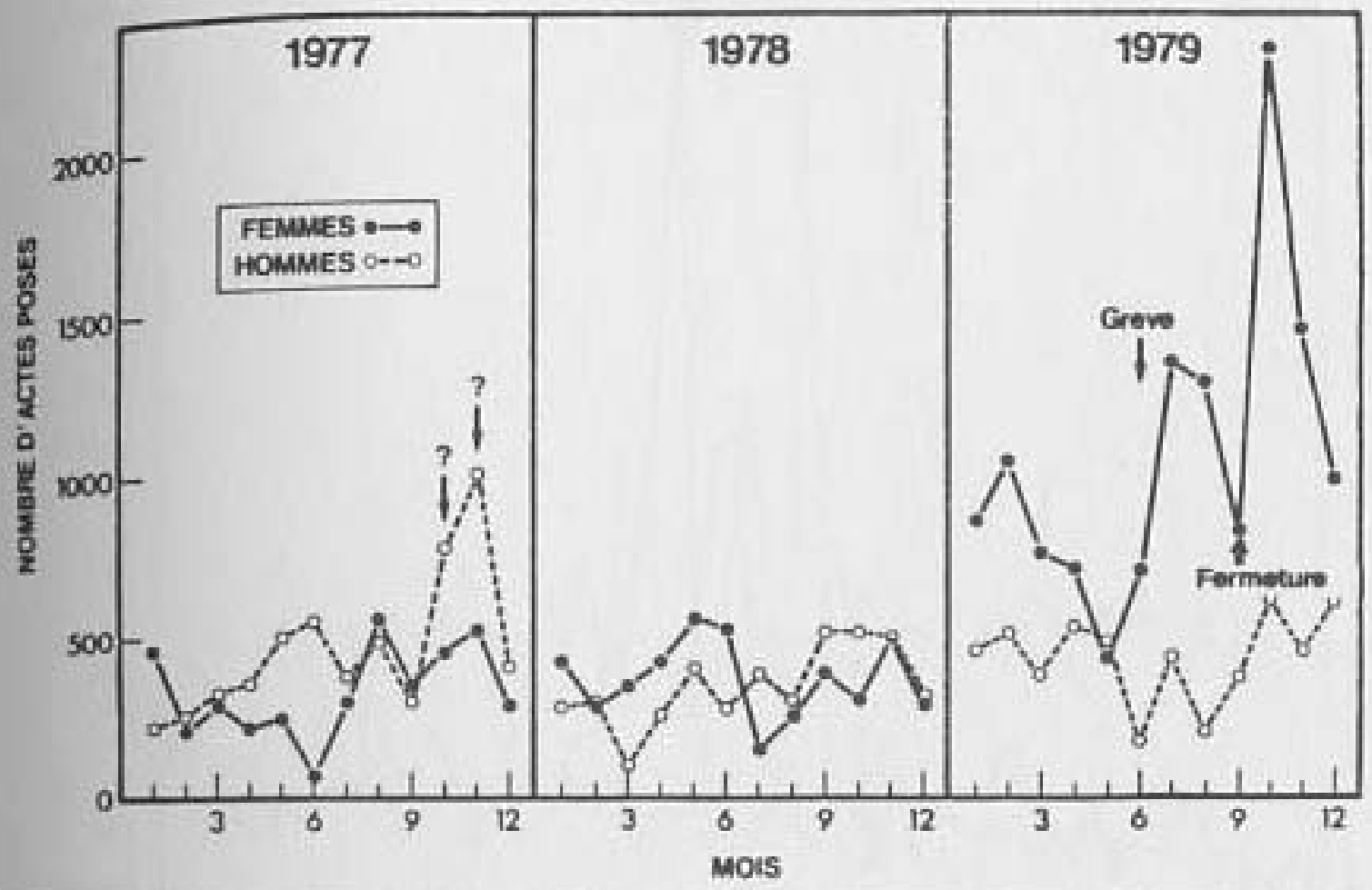

tion, dépistage de cancer du sein, etc.) ne s'est produit à cette période (octobre 1979) à notre connaissance. En ce qui a trait au supra-régional, l'ajout d'une région ćconomique témoin s'avérait le moyen le plus efficace d'écarter l'hypothèse d'un événement national: la spécificité du phénomène à la région de Port-Cartier, vis-à-vis cette région témoin élimine, en grande partie, cette menace à la validité de conclusion. La région de Jonquière (Québec) fut retenue à cause d'une vocation économique et d'une situation géographique comparables sur bien des points à celles de la région de notre étude.

Un premier examen visuel des données enregistrées (réelles) chez les femmes de 30-39 ans (examens complets seulement) indique lie la distribution des données, sauf pour figuration et est de même niveau dans les deux régions. Bouffées et affaissements se sucoedent à chaque période de 4-6 mois; il y a Ėalement une tendance linéaire positive (augmentation légère mais régulière du mois 1 à 58), (figure 2). On remarque que dans la région de Port-Cartier il $y$ a de fortes augmentations au 3 lième et 32 ième mois d'abord (après le début de la grève) et, une autre très importante celle-là, au 34ième mois, (i.e. 6 mois suivant la fermeture). II faut toutefois noter que les premières augmentations suivent le patron déjà présent en 1978 (mois 17 et 18 ).

L'analyse de la matrice d'autocorrélations révèle la présence d'une configuration redondante dans les données mensuelles; les paramètres de cette configuration sont les mêmes pour les deux régions (moyenne mouvante, ordre 1 , cycle sur 12 mois). Pour Jonquière, le $x^{2}$ de l'analyse de la matrice est de 97,12 ( $p<.001$; d1,25) et pour PortCartier il est de $46,00(p<.01 ;$ dI, 25$)$. Ces $\mathrm{X}^{2}$ deviennent non-significatifs lorsque l'on déleste les données réelles des données générées (prédites) par le modèle de configuration retenu (Jonquière: 24:83; PortCartier: 9:10). Cette dernière analyse confirme la validité du modêle. D'ailleurs, on constate le recouvrement quasi-parfait entre données réelles et prédites dans la région de 


\section{Figure 2}

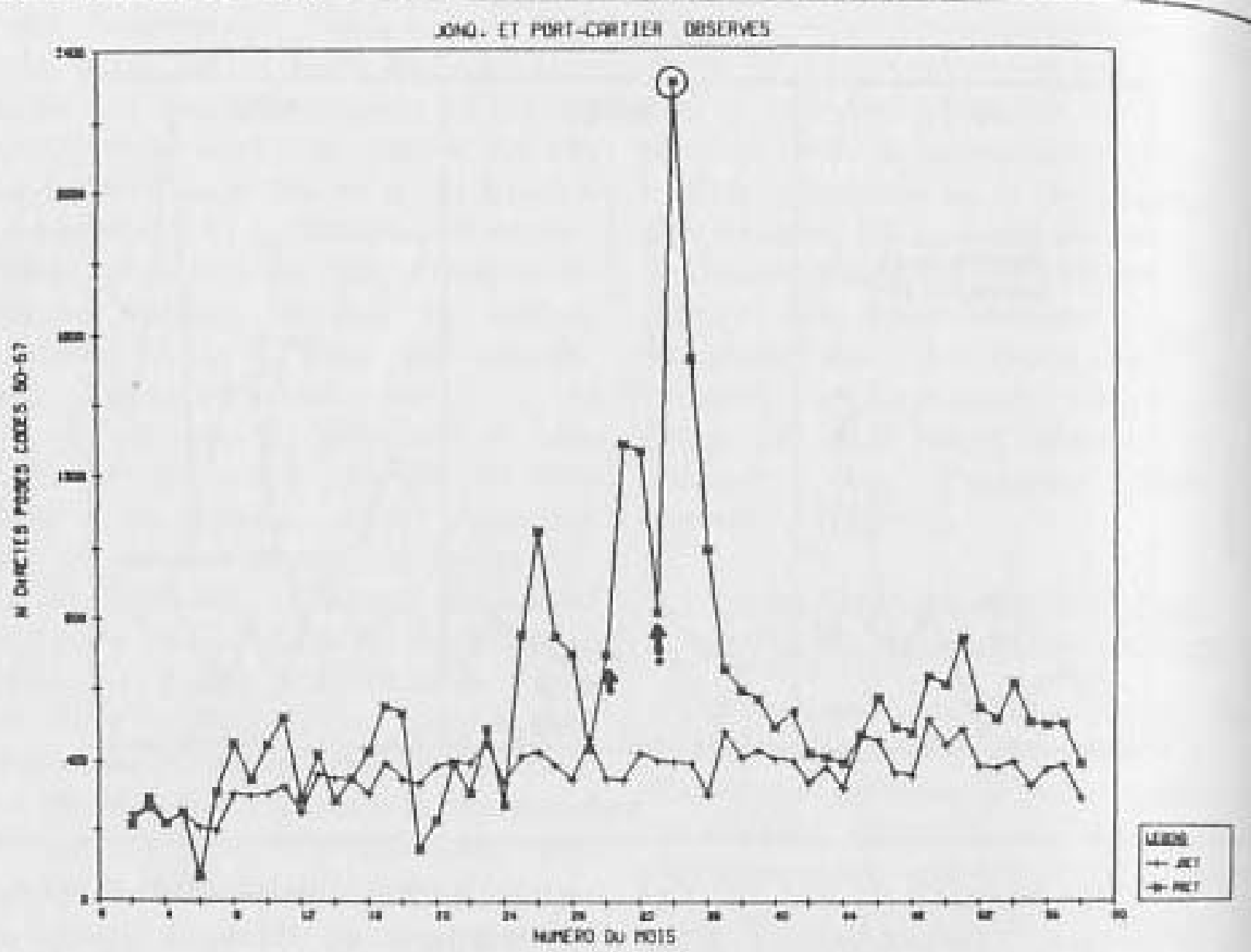

Jonquière. Pour ce que est de la région de Port-Cartier, on note un recouvrement également important. Seule la donnée réelle enregistrée au 34ième mois s'écarte sensiblement et clairement de la donnée prédite par le modèle. Cet écart s'exprime avec encore plus de force lorsque l'on examine les courbes de scores résiduels (figure 3 ). On ne retrouve une telle bouffée qu'au 34ième mois de la série de Port-Cartier.

\section{DISCUSSION}

La méthodologie employée (i.e, utilisation d'une région témoin et emploi de statistiques sur séries chronologiques parallèles de 58 points) permet de suggérer l'existence d'un lien spécifique entre la fermeture de l'usine Rayonnier-Québec à Port-Cartier et une augmentation des examens complets chez ce groupe de femmes de $30-39$ ans dans cette région.

Reconnaitre l'existence de ce lien specifi- que force à avancer quelques explications du phénomène. L'hypothèse retenue s' inspire du modèle dit de "l'amortissement du stress," ("Stress buffering") (Dean et Lin, 1977; Dooley et Catalano, 1981; Gore, 1978; Liem et Liem, 1978). Essentiellement, ce modele avance que le stress et ses manifestations seront amortis à la mesure des ressources personnelles et du support social des individus. Dans le cas présent, les femmes de 30-39 ans constitueraient le groupe éprouvant le pless grand stress. Sans doute majoritairenent mères d'enfants d'âge scolaire ou pre scolaire, elles forment un groupe vulnerable (Brown, 1975); leur statut et responsabilities les tiendraient à l'écart d'une participation active à la solution du problème, et de sources de support affectif, informatif of récrẻatif; cet accès serait disponible aut hommes, de par leur milieu de travail, de loisirs ou d'associations.

L'exclusivité de l'augmentation des es 
Figure 3

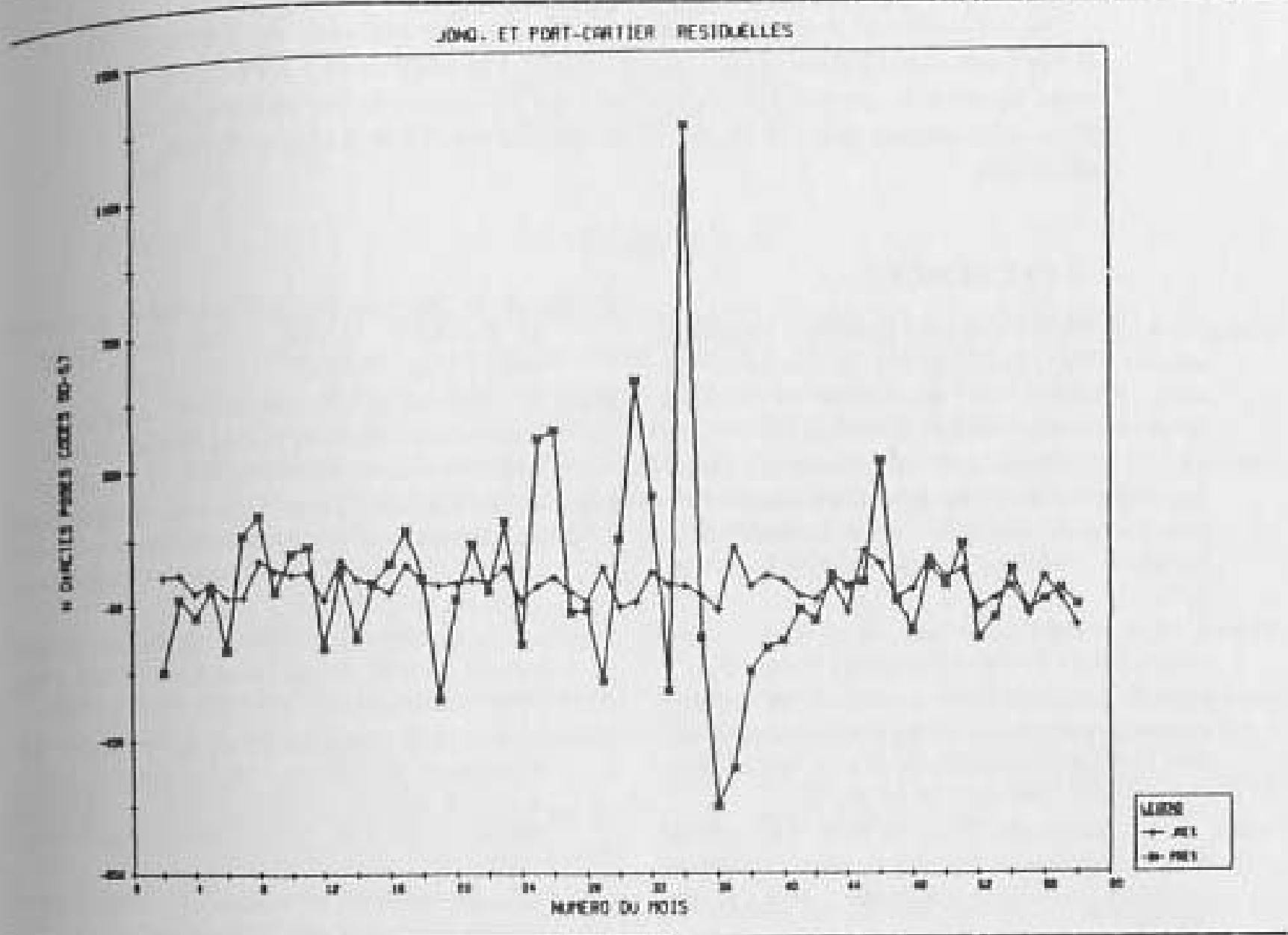

amens complets chez les femmes de 30-39 ans, l'inexistence de ce phènomène chez les hommes (toutes strates d'âges) vient appuyer cette hypothèse. D'autres données disponibles (Bouchard et Perreault, 1981) encouragent à une telle interprétation; il $y$ a stabilisation des fréquences de diagnostics psychophysiologiques chez les hommes en 1979, immédiatement après la fermeture, et non chez les femmes. D'autre part, l'enquête menéc auprès des informateurs-clés suggère une perturbation de l'environnement des femmes de $30-39$ ans: augmentation sentie de ta violence familiale, de l'alcoolisme massculin, de séparations, de divorces, de diffícultés scolaires, etc. D'un autre côté, constatations à l'effet de l'expérience positive de sette fermeture chez les femmes participant à des groupes d'entraide: nouvelles amitiés, nouvelles orientations de carrière, satisfaction à contribuer à la solution des problèmes, distraction des problèmes personnels, etc.
Les obligations et le rỏle des femmes de 30-39 ans les eloignent sans doute de telles stratégies d'adaptation.

Il va de soi que l'hypothèse avancée ici demande vérification au moyen d'études prospectives approfondies et contrôlées. Une étude rétrospective telle celle rapportẻe ici ne peut contribuer crédiblement à l'élaboration d'un modẻle explicatif. Toutefois, la stratégie employée permet d'isoler, de définir et de prévoir l'impact d'événements semblables; elle peut s'avérer un outil utile (et peu coûteux) à la réflexion de celles et ceux qui souhaitent des changements radicaux dans la gestion d'une "économie en folie" (Catalano, 198). Force nous est de reconnaître le peu de recherche dans un domaine où l'accès à des données demeure, tous comptes faits, relativement aisé et grandement instructif. 


\begin{abstract}
The utilization of medical services is analysed before and after the closing of the Rayonnier-Québec plant in Port Cartier. The analysis of a 5 year time series suggests a specific association between the plant closing and the increase of complete physical examinations among women in the 30 to 39 year age group.
\end{abstract}

\section{RÉFÉRENCES}

Adams, O.B. Heath and economic acrivdy: A pimessies analysis of Canadian morrality and unemployment nates. Ministère des equipements of services, Gouvernement du Canada, décembre 1981.

Bouchard, C, et Perreault, R. Fermeture de Pusine Rayonnier-Quebec ef taux de consultations medi. cales par les cifoyens et citoyennes de Part-Cartier. Document technique inédit, LAREHS, janvier 1981.

Brenner, M.H. Mental illites and the rconomy. Cambridge, Mass,: Harvard University, Press, 1973.

Brenuer, M.H. Estimiating the social costs of national economy policy: Implications for mental and phy. sical health and criminal aggression. Washington, D.C., Government printing office, 1976.

Brown, G.W + Bhrolcrain, M.N, et Harris, T. "Social class and psychiatric disturbance among women of at urban population. Sociology, 1975, 9, pp. 225-254.

Catalano, R. Psychology amid economic madness: an economist's advice for the $80^{\prime}$ s. Conferrence presentée au 1 lième Congrès de l'Association scientifique pour la modification du comporiement, Montréal, mai 1980.

Dean, A. et Lin, N. The stress buffering role of social support. The Journal of Nervois and Mental Diseaser, 1977, 165, pp. 403.417.
Dooley, D. et Cataluno, R. Economic change as a calle of behavioral disorder. Psychological Bullerie $1980,87, \mathrm{pp}, 450-469$.

Gore, $S$. The effect of social support in moderating tie health consequences of unemployment. Journat of Health and Social Behavior, 1979, 19, pp. 157.169,

Liem, R. et Liem, 1. Social class and mental heals reconsidered; the role of economic stress and socia support. Joumal of Health and Social Beharior. $1978,19, \mathrm{pp}, 139-156$,

Steinberg, L, Catalano, R. et Dooley, D. Econotnic anis. cedents of child abuse and neglect. Child fend opment, 1981, 52, pp. 975-985.

Vigderhous, G. Forecasting sociological phenomena: $A$ p plication of Box-Jenkins methodology to sticide rates. In Kart F. Schuessler (Ed.) Sociolopica Methodology 1978. San Francixco: Josiey Bain, 1977.

Cyclical variations of monthly and yearly bomicide rates in the United States and their relationship to changes in the unemployment rale. in Charles Wellford (Ed.) Quantitative studied is criminalogy (Vol z), Beverley Hill: Sage Publications, 1978:

Yigderhous, G. et Fishman,G. "The impact of unemploy. ment and familial integration on changing sucide rates in U.S.A., 1920-1969, Soctal Psychiarm, 1976,13, pp. 239-248. 